\title{
Electron-Optical Sectioning for Three-Dimensional Imaging of Crystal Defect Structures
}

\author{
Peter D. Nellist \\ Department of Materials, University of Oxford, United Kingdom \\ peter.nellist@materials.ox.ac.uk
}

\begin{abstract}
The depth of field of an optical imaging system is proportional to the inverse square of the numerical aperture. The development of electron-optical devices to correct for the inherent spherical aberration of electron optics has led to a dramatic increase in numerical aperture that therefore also result in dramatic reductions in depth of field. The depth of field of a state-of-the-art system may now reach below $5 \mathrm{~nm}$. An opportunity is therefore created to measure three-dimensional information about a sample by focusing on specific layers within the sample, a process known as optical sectioning. In this short review, we examine some of the properties of the technique, and illustrate its use with a range of applications to semiconducting materials that have been presented in the literature.
\end{abstract}




\section{Introduction}

The development of aberration correctors in transmission electron microscopy has led to major advances in resolution and contrast both in conventional images and spectroscopic maps [1-4]. In the case of the scanning transmission electron microscope (STEM), the increased numerical aperture (NA) of the objective lens (OL) allowed by the use of aberration correctors leads to a smaller, more intense illuminating electron probe which is the origin of the improved performance. The increased numerical aperture, however, also leads to a reduction in the depth of field (DOF). Optics texts will often refer to the DOF as being inversely proportional to the square of the numerical aperture. The actual situation is somewhat more complicated and a full discussion requires consideration of the three-dimensional (3D) transfer function. Nonetheless, the DOF is very sensitive to the NA of the OL, and improvements in aberration-correction technology are leading to continual reductions in the DOF.

The reduction in DOF may be regarded as being detrimental to image interpretation if it is being assumed that the image represents a projection of the sample. In a state-of-the-art instrument the DOF may be below $5 \mathrm{~nm}$ - less than the typical thickness of a sample. An opportunity is created, however, to use the reduced DOF to investigate specific depths within the sample - relying on the out-of-focus regions not to strongly contribute to image contrast. This process is known as "optical sectioning".

Here we aim to review the application of optical sectioning to the detection of defects, strain fields and the structure of dislocations and in particular dislocation cores, in semiconductor materials. Optical sectioning can be achieved using annular dark-field (ADF) imaging in STEM, but in order to interpret the contrast, we must also consider the form of the 3D transfer function that applies. We start by highlighting some of the initial experimental results in optical sectioning, before considering how these can be interpreted in terms of the transfer function. We then go on to review the application of optical sectioning to detecting the 3D position of dopant atoms, measuring the depthdependent strain in a sample, and the local structure of dislocations.

\section{Initial experimental results in optical sectioning}

Early applications of optical sectioning in ADF STEM came from the Pennycook group at Oak Ridge National Laboratory making use of annular dark-field STEM. A schematic of the ADF STEM experiment is shown in Fig. 1. Details of the properties of ADF STEM are described in more detail in [5], but briefly the high-angle scattering leads to an image that is strongly dependent on atomic number, Z, and the large extent of the detector leads to the image being regarded as incoherent which will be explained in more detail later.

Figure 2 shows ADF STEM images from a focal series showing the gate region of a high- $k$ dielectric device presented by Van Benthem et al. [6]. Single $\mathrm{Hf}$ atoms in the $\mathrm{SiO}_{2}$ layer can be seen to become visible, and then disappear as the focus is changed. Using this approach the height of a number of $\mathrm{Hf}$ atoms could be determined. The authors noted that the $\mathrm{Hf}$ atoms were only visible over a range of 1.5-2.5 nm. Although the authors do not quote the beam energy and numerical aperture of their microscope, they do note that the depth of focus can be approximated to

$$
\Delta z \sim \frac{\lambda}{\alpha^{2}}
$$


(1)

where $\lambda$ is the electron wavelength and $\alpha$ the semi-angle of convergence of the probe-forming beam (equivalent to the NA since $\alpha$ is small) and they mention that their depth resolution would be about $6.6 \mathrm{~nm}$. They ascribe the smaller defocus variation over which the atoms were visible as arising because of the background signal and the signal-to-noise of their experiment.

This initial work was followed up with the application of optical sectioning to heterogeneous catalyst samples by Borisevic et al. [7]. The aim of the work was to detect the 3D configuration of the catalyst nanoparticles with respect to the support material. In that paper they note that the depth resolution achieved does not appear to match that given by Eq. (1). This observation was followed up more systematically by Behan et al. [8] (Fig. 3) who found that the intensity of an nanoparticle in a 3D stack of images recorded in a focal series showed a depth profile with a full-width at halfmaximum of hundreds of nanometres. They showed that for a feature of diameter, $d$, the integrated intensity of the scattering from that feature would only start to show a decrease once the electron probe was sufficiently defocus that it had spread wider than the feature. Mathematically this can be written as

$$
\Delta z=\frac{d}{\alpha}
$$

Assuming a nanoparticle diameter of $3.8 \mathrm{~nm}$ and a beam convergence semi-angle, $\alpha$, of $22 \mathrm{mrad}$ results in an effective depth resolution of $172 \mathrm{~nm}$, which corresponds well to the data shown in Refs [8] and [7] and is too large to be of much use for thin TEM samples.

That the depth resolution depended on the lateral extent of the object was already known in the light-optics literature [9], but of course the much larger numerical apertures available for light optics lessons the deleterious impact of this result somewhat.

\section{The 3D transfer function}

By analogy with the light-optics literature [9], we can explore the full 3D optical transfer function (OTF) for the optical sectioning approach. The OTF is plotted in reciprocal space and is an expression of the strength of transfer to an image as a function of spatial frequency. For a an object lying at a distance, $z$, from the focal plane of the microscope, and assuming the incoherent approximation, the image can be written as a convolution

$$
I(\boldsymbol{R})=P(\boldsymbol{R}, z) \otimes O(\boldsymbol{R})
$$

Where $P(\boldsymbol{R}, z)$ is the intensity of the illuminating probe as a function of lateral position, $\boldsymbol{R}$, for a microscope defocus of $z$, and $O(R)$ is an object function that represents the scattering to the detector. Treating $P(\boldsymbol{R}, z)$ as a 3D plot of the intensity in the STEM probe, the OTF can then be calculated by taking the 3D Fourier transform of $P(\boldsymbol{R}, z)$. The result, taken from [8], is shown in Fig. 4. A similar result was also shown by Intaraprasonk et al. [10]. 
The striking feature of the OTF is the large missing cone. For spatial frequencies within this missing cone, no information will be transferred to the image. It can be seen that for low lateral spatial frequencies, the missing cone only allows low longitudinal spatial frequencies to be transferred. Thus low lateral spatial frequencies have poor depth resolution, confirming the result in Eq. (2). A confocal optical configuration can act to fill the missing cone in the transfer function, indeed this is a prime motivation for confocal microscopy. Experimental implementation of SCEM has been demonstrated [11-13]. The most effective mode in SCEM is energy-filtered SCEM, but this is hampered by the residual chromatic aberration of the optics [11]. Information outside the bounds of transfer can be reconstructed through the use of prior information, such as in a deconvolution process [14], though care must be taken over the generation of artefacts using such approaches.

If we instead focus on applications where high-lateral spatial frequencies are present, which would correspond to atomic resolution imaging, then Fig. 4 shows that high-depth resolutions can be achieved, and this is the approach used for the applications shown in the rest of this review. To summarise this section in a simple way: high-resolution images are much more sensitive to focus than lower-resolution ones, and therefore provide the highest depth resolution for optical sectioning.

\section{Detection of individual dopant atoms and the influence of channelling}

The analysis presented in Section 3 ignores the effect of scattering of the incoming electron beam by the sample before it has had chance to converge into a focused probe. In particular, when a crystalline material is imaged along a low-order zone-axis in order to form an atomic-resolution image, the attractive atomic potential of the atoms along an atomic column cause the probe to channel along the column. The effect is somewhat similar to a wave in a waveguide, and oscillations in intensity known as pendellösung are seen. Cosgriff and Nellist [15] have analysed the process of optical sectioning in the presence of channelling using a Bloch wave approach. Three important effects are seen: (i) The higher-angle rays included in the convergent beam formed with aberrationcorrected optics have a much higher excitation error and therefore interact in a rather more kinematical way with less channelling (which can be regarded as a dynamical effect). (ii) The channelling oscillations modulate the formation of the depth-dependent peak in intensity of the probe in the crystal (Fig. 5), leading to the possibility that a dopant atom may be located at a minimum of a channelling oscillation and may therefore not be detected. (iii) The potential of the atomic column leads to an additional focusing effect that bring the beam to a focused intensity maximum approximately $2 \mathrm{~nm}$ before the set defocus value.

Comprehensive simulations of these effects have been performed by Xin et al. [16] (Fig. 6). They showed that in an amorphous matrix, a heavy impurity atom can be detected with a maximum intensity when the depth-dependent intensity peaks at the atom site. In a crystalline matrix, intensity maxima are seen when the probe is focused at the entrance surface, and with the defocus just beyond the impurity atom size (showing that the pre-focusing effect of the column is present). They also showed that for certain depths, the impurity atom is hard to detect, presumably because it lies in a channelling minimum. Similar effects are also seen for depth-dependent spectroscopic mapping of impurities [17]. Using low-angle ADF, peaks in a depth scan are also seen at interfaces perpendicular to the beam in heterostructured samples [18]. 
Thus for single impurity atom detection, the effect of channelling complicates their detection. Indeed, channelling alone has been used to provide a sensitivity to depth without the use of a focalseries $[19,20]$. Recently, an approach has been proposed to combine focal series with changes in convergence angle [21], with the latter having the effect of adjusting the channelling oscillation frequency and thereby moving the positions of the minima. Further control of the illumination beam in this way appears to be a useful way to provide data that contains useful depth information when channelling is present. Xin et al. [16] also suggest that tilting the sample away from channelling conditions will provide simpler to interpret data, albeit with the loss of atomic resolution imaging.

\section{Detection of depth-dependent strain}

Whilst a single impurity atom is a highly localised defect, displacements due to strain are usually longer range in nature. Strain plays an important role in controlling carrier mobility in semiconducting devices, and Kim et al. [22] have shown that a depth-dependent strain can be measured in the vicinity of a strain channel in the transistor. A decrease in the strain field towards the silicon substrate was revealed. A cross-section sample was used to verify the depth-dependent measurements.

The detection of depth-dependent strain has also been used to detect the existence of a screw component in dislocations viewed end-on at high resolution. When dislocations with a screw component emerge at the surface of a thin foil prepared for TEM observation, a surface relaxation known as the Eshelby twist occurs [23]. The Eshelby twist is a depth-dependent counter-rotation of the lattice penetrating from the two surfaces of the thin foil. Using optical sectioning to measure the depth-dependent rotation of the image, Lozano et al. [24] were able to detect the presence and sign of the screw component of dislocations viewed along the [0001] in GaN. In particular, this led to the unambiguous identification of mixed $\mathbf{b}=[\mathbf{a}+\mathbf{c}]$ dislocations in GaN (Fig. 7).

\section{Optical sectioning of dislocation core structures}

The identification of mixed $[\mathrm{a}+\mathrm{c}]$ dislocations in $\mathrm{GaN}$ by detecting depth-dependent strain allowed the core of these dislocations to be examined at high-resolution. It was found that in the sample analysed, the mixed $[\mathbf{a}+\mathbf{c}]$ dislocations showed a dissociated core structure. Direct observation of the transverse (edge) displacements showed that the edge [a] component had dissociated into two partial dislocations with edge component $[1 / 2 \mathbf{a}]$. The overall $[\mathbf{a}+\mathbf{c}]$ dislocations were also found to be inclined to the [0001] direction, therefore containing a relatively high density of kinks and jogs. The reduction in depth of focus arising enable a clear image of the dislocation core to be formed, as shown in Fig. 7, despite the dislocation inclination. Making the assumption that the screw component of the $[\mathbf{a}+\mathbf{c}]$ had also dissociated such that the two partials were each of $[1 / 2 \mathbf{a}+1 / 2 \mathbf{c}]$ type, and including some kinks within the depth of field of the image, a good match to the image peaks could be made [25].

The presence of kinks in a Cd-core $90^{\circ}$ partial dislocation in CdTe has also been observed using optical sectioning by Li et al. [26] (Fig. 8). In a focal series of images, translation of the dislocation can be observed between particular pairs of images, with the dislocation core remaining stable between the others. The depth of kinks in the dislocation could thereby be determined using this approach. 
Returning to the dissociated $[\mathbf{a}+\mathbf{c}]$ dislocation in $\mathrm{GaN}$, the dissociation reaction of the screw component into [ $1 / 2 c]$ components on each partial dislocation was proposed in [25] through energetic considerations associated with the lack of wrong bonds in the resultant fault between the partial dislocations. This motivated the idea of using optical sectioning to directly image the screw displacements associated with this dislocation structure.

It has been shown that screw displacements in materials can be directly observed using ADF STEM with the dislocation lying transverse to the incident beam direction, but this observation required the use of a material where the screw dislocation Burgers vector was extremely large. In Li et al. [27], the material used was a novel $\mathrm{HfAl}_{4} \mathrm{C}_{4}$ ceramic containing a screw dislocation with a burgers vector length of $2.19 \mathrm{~nm}$. In the case of the dissociated GaN mixed dislocation described above, the smaller screw burgers vector component of $0.26 \mathrm{~nm}$ of the proposed partial dislocation was found to need optical sectioning in order to detect it. In Fig. 9, an image is shown with the focal plane set just before the plane containing a dissociated $[\mathbf{a}+\mathbf{c}]$ dislocation. Although the image was recorded along a low-order zone axis (in this case the crystallographic a axis), and despite the effects of channelling, the image is still relatively simple to interpret. As described in [28], the finite depth of field allows the atomic locations both just before and just after the plane containing the dislocation to be imaged. From these types of images, the helicoidal nature of the atomic displacements around the dislocation core can be directly imaged. A row of bright peaks followed across the dislocation line is seen to shear by a distance of half the screw component of the burgers vector, and similarly a row of weaker peaks is seen to shear in the opposite direction also by a distance of half the screw component of the burgers vector. In the case of the dissociated [a+c] dislocation (Fig. 9), two stages of shear can be seen, each of a distance of $[1 / 4 \mathbf{c}]$ thus indicating the partials with burgers vector $[1 / 2 c]$. The proposed dissociation reaction of the $[\mathbf{a}+\mathbf{c}]$ dislocation into two $[1 / 2 \mathbf{a}+1 / 2 \mathbf{c}]$ partial dislocations was therefore confirmed using optical sectioning, and a detailed image analysis allowed a dissociation width of $1.65 \mathrm{~nm}$ to be measured. This narrow fault width could not be detected using earlier methods such as weak-beam imaging.

\section{Conclusions}

Here we have reviewed current status of the emerging field of optical sectioning in ADF STEM. From the work performed to date, it is clear that depth determination of single impurity atoms through collection of a focal-series of images, or through use of channelling oscillations alone, has limitations. These might be overcome through imaginative variation of other imaging parameters, such as convergence angle.

More promising so far experimentally is the detection of depth-dependent strain. In particular the displacement of atoms associated with dislocation cores can be examined with greater precision using optical sectioning. This approach has led to the identification of a hitherto unknown dissociation reaction of mixed dislocations in GaN.

It is striking that the actual demonstrations of optical sectioning in ADF STEM have so far all been to semiconductor materials. This is perhaps indicative of the importance of both the configuration of dopant atoms and dislocation structures in these materials. There are, however, many other materials classes where the 3D capabilities of optical sectioning can play an important role. For example, screw dislocations in body-centred cubic metals show poor mobility that can restrict the ductility of these materials. It has been suggested that this poor mobility is due to a delocalisation of 
the dislocation core. Although this effect has been widely modelled, to date there is no incontrovertible experimental evidence for this effect. An important hindrance has been the Eshelby twist associated with the screw dislocation. It has been suggested that the use of optical sectioning to image below the Eshelby twist may offer a route to directly image the delocalisation of the dislocation core in this case [29].

The inverse square dependence of the depth of field on numerical aperture means that as STEM aberration correction technology improves, the depth of field will rapidly reduce. Ishikawa et. al [30] have discussed the impact of larger convergence angle, and have discussed that $100 \mathrm{mrad}$ convergence at $300 \mathrm{keV}$ beam energy brings the DOF to within the unit cell dimensions of strontium titanate $(0.4 \mathrm{~nm})$. There continues to be developments in the geometric correctors which allow higher angles. Chromatic aberrations are more challenging to correct, but either hardware correctors or monochromators can play a role here. The emergence of these technologies suggest that optical sectioning will be a technique of increasing importance.

\section{Acknowledgements}

PDN acknowledges the Leverhulme trust and the EPSRC for funding associated with electron-optical sectioning research. 


\section{References}

[1] O.L. Krivanek, N. Dellby, A.R. Lupini, Towards sub-Å electron beams, Ultramicroscopy, 78 (1999) $1-11$.

[2] M. Haider, H. Rose, S. Uhlemann, E. Schwan, B. Kabius, K. Urban, A spherical-aberrationcorrected 200kV transmission electron microscope, Ultramicroscopy, 75 (1998) 53-60.

[3] P.D. Nellist, M.F. Chisholm, N. Dellby, O.L. Krivanek, M.F. Murfitt, Z. Szilagyi, A.R. Lupini, A. Borisevich, W.H.J. Sides, S.J. Pennycook, Direct Sub-Angstrom Imaging of a Crystal Lattice, Science, 305 (2004) 1741.

[4] D.A. Muller, L.F. Kourkoutis, M. Murfitt, J.H. Song, H.Y. Hwang, J. Silcox, N. Dellby, O.L. Krivanek, Atomic-scale chemical imaging of composition and bonding by aberration-corrected microscopy, Science, 319 (2008) 1073-1076.

[5] P.D. Nellist, S.J. Pennycook, The Principles and Interpretation of Annular Dark-Field Z-Contrast Imaging, Advances in Imaging and Electron Physics, 113 (2000) 148-203.

[6] K. Van Benthem, A.R. Lupini, M. Kim, H.S. Baik, S. Doh, J.-H. Lee, M.P. Oxley, S.D. Findlay, L.J. Allen, J.T. Luck, S.J. Pennycook, Three-dimensional imaging of individual hafnium atoms inside a semiconductor device, Appl. Phys. Lett., 87 (2005) 034104.

[7] A.Y. Borisevich, A.R. Lupini, S.J. Pennycook, Depth sectioning with the aberration-corrected scanning transmission electron microscope, Proceedings of the National Academy of Sciences, 103 (2006) 3044-3048.

[8] G. Behan, E.C. Cosgriff, A.I. Kirkland, P.D. Nellist, Three-dimensional imaging by optical sectioning in the aberration-corrected scanning transmission electron microscope, Philosophical Transactions of the Royal Society of London A, 367 (2009) 3825-3844.

[9] B.R. Frieden, Optical transfer of the three-dimensional object, Journal of the Optical Society of America, 57 (1967) 36-41.

[10] V. Intaraprasonk, H.L. Xin, D.A. Muller, Analytic derivation of optimal imaging conditions for incoherent imaging in aberration-corrected electron microscopes, Ultramicroscopy, 108 (2008) 1454-1466.

[11] P. Wang, G. Behan, M. Takeguchi, A. Hashimoto, K. Mitsuishi, M. Shimojo, A.I. Kirkland, P.D. Nellist, Nanoscale Energy-Filtered Scanning Confocal Electron Microscopy Using a DoubleAberration-Corrected Transmission Electron Microscope, Physical Review Letters, 104 (2010) 200801.

[12] P. Wang, A. Hashimoto, M. Takeguchi, K. Mitsuishi, M. Shimojo, Y. Zhu, M. Okuda, A.I. Kirkland, P.D. Nellist, Three-dimensional elemental mapping of hollow $\mathrm{Fe}_{2} \mathrm{O}_{3} @ \mathrm{SiO}_{2}$ mesoporous spheres using scanning confocal electron microscopy, Appl. Phys. Lett., 100 (2012) 213117.

[13] A. Hashimoto, M. Shimojo, M. Takeguchi, Three-dimensional imaging of carbon nanostructures by scanning confocal electron microscopy, Journal of Applied Physics, 106 (2009) 086101.

[14] R. Ramachandra, N. de Jonge, Optimized Deconvolution for Maximum Axial Resolution in ThreeDimensional Aberration-Corrected Scanning Transmission Electron Microscopy, Microscopy and Microanalysis, 18 (2012) 218-228.

[15] E.C. Cosgriff, P.D. Nellist, A Bloch wave analysis of optical sectioning in aberration-corrected STEM, Ultramicroscopy, 107 (2007) 626-634.

[16] H.L. Xin, V. Intaraprasonk, D.A. Muller, Depth sectioning of individual dopant atoms with aberration-corrected scanning transmission electron microscopy, Appl. Phys. Lett., 92 (2008) 013125.

[17] A.J. D'Alfonso, S.D. Findlay, M.P. Oxley, S.J. Pennycook, K. van Benthem, L.J. Allen, Depth sectioning in scanning transmission electron microscopy based on core-loss spectroscopy, Ultramicroscopy, 108 (2007) 17-28.

[18] G. Ruben, E.C. Cosgriff, A.J. D'Alfonso, S.D. Findlay, J.M. LeBeau, L.J. Allen, Interface location by depth sectioning using a low-angle annular dark field detector, Ultramicroscopy, 113 (2012) 131-138. [19] P.M. Voyles, D.A. Muller, E.J. Kirkland, Depth-dependent imaging of individual dopant atoms in silicon, Microscopy and Microanalysis, 10 (2004) 291-300. 
[20] R. Ishikawa, A.R. Lupini, S.D. Findlay, T. Taniguchi, S.J. Pennycook, Three-Dimensional Location of a Single Dopant with Atomic Precision by Aberration-Corrected Scanning Transmission Electron Microscopy, Nano Letters, 14 (2014) 1903-1908.

[21] E. Rotunno, M. Albrecht, T. Markurt, T. Remmele, V. Grillo, Three dimensional analysis of the composition in solid alloys by variable probe in scanning transmission electron microscopy, Ultramicroscopy, 146 (2014) 62-70.

[22] S. Kim, Y. Jung, S. Lee, J. Jung Kim, G. Byun, S. Lee, H. Lee, 3D strain measurement in electronic devices using through-focal annular dark-field imaging, Ultramicroscopy, 146 (2014) 1-5.

[23] J.D. Eshelby, A.N. Stroh, Dislocations in thin plates, Philosophical Magazine Series 7, 42 (1951) 1401-1405.

[24] J.G. Lozano, H. Yang, M.P. Guerrero-Lebrero, A.J. D'Alfonso, A. Yasuhara, E. Okunishi, S. Zhang, C.J. Humphreys, L.J. Allen, P.L. Galindo, P.B. Hirsch, P.D. Nellist, Direct Observation of DepthDependent Atomic Displacements Associated with Dislocations in Gallium Nitride, Physical Review Letters, 113 (2014) 135503.

[25] P.B. Hirsch, J.G. Lozano, S. Rhode, M.K. Horton, M.A. Moram, S. Zhang, M.J. Kappers, C.J. Humphreys, A. Yasuhara, E. Okunishi, P.D. Nellist, The dissociation of the [a $+\mathrm{c}]$ dislocation in GaN, Philosophical Magazine, 93 (2013) 3925-3938.

[26] C. Li, J. Poplawsky, Y. Wu, A.R. Lupini, A. Mouti, D.N. Leonard, N. Paudel, K. Jones, W. Yin, M. AlJassim, Y. Yan, S.J. Pennycook, From atomic structure to photovoltaic properties in CdTe solar cells, Ultramicroscopy, 134 (2013) 113-125.

[27] F. Li, H. Nian, K. Du, J. Wang, J. Wang, Y. Zhou, Direct Observation of a Screw Dislocation Normal to the Beam by Z-Contrast STEM, Journal of the American Ceramic Society, 95 (2012) 466-468.

[28] H. Yang, J.G. Lozano, T.J. Pennycook, L. Jones, P.B. Hirsch, P.D. Nellist, Imaging screw dislocations at atomic resolution by aberration-corrected electron optical sectioning, Nature Communications, 6 (2015) 7266.

[29] E.C. Cosgriff, P.D. Nellist, P.B. Hirsch, Z. Zhou, D.J.H. Cockayne, ADF STEM imaging of screw dislocations viewed end-on, Philosophical Magazine, 90 (2010) 4361-4375.

[30] R. Ishikawa, A.R. Lupini, Y. Hinuma, S.J. Pennycook, Large-angle illumination STEM: Toward three-dimensional atom-by-atom imaging, Ultramicroscopy, 151 (2015) 122-129. 
Figure 1. A cross-sectional schematic of the scanning transmission electron microscope. The dashed ray paths show how the probe is scanned across the sample. High-angle scattering is detected by an annular detector as marked.

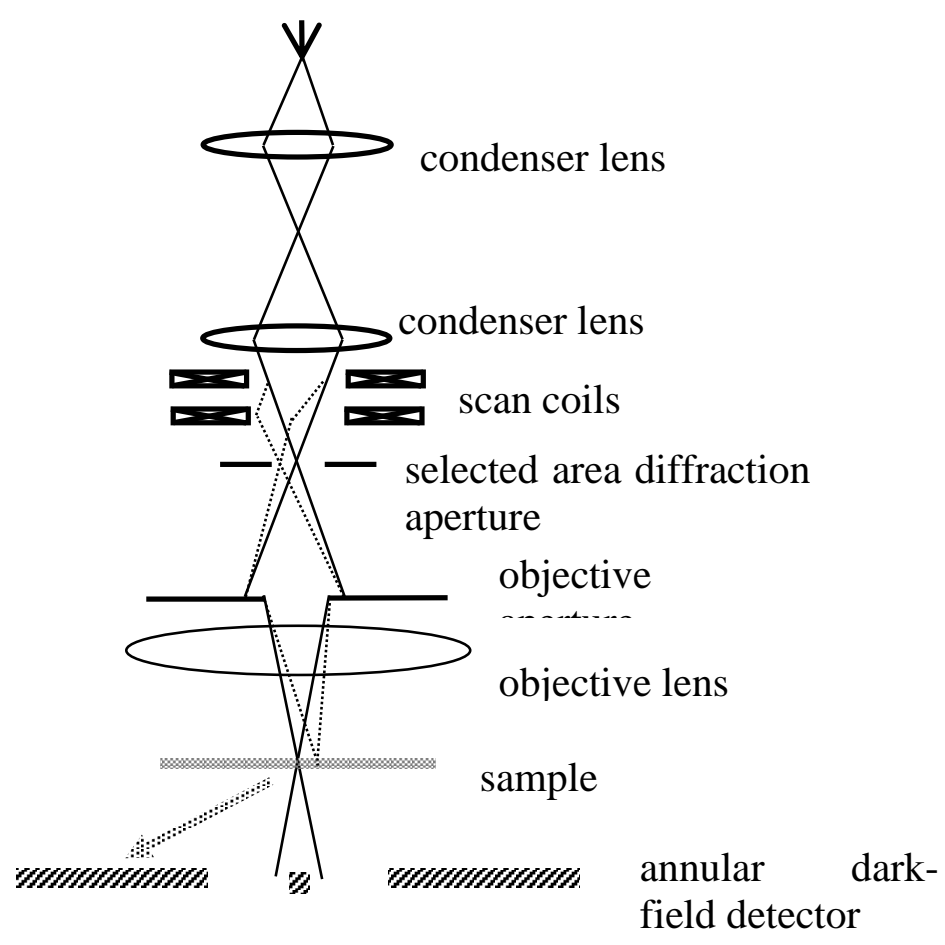


Figure 2. Sections from an 3D ADF STEM image stack of a gate-oxide structure. The bright region is a $\mathrm{HfO}_{2}$ film. A single $\mathrm{Hf}$ atom can be seen in the amorphous $\mathrm{SiO}_{2}$ interlayer, with the $\mathrm{Si}$ lattice seen to the right of the image. The top left image is an z-y section of the image stack at a defocus where the $\mathrm{Hf}$ appears. The right and bottom images show an $y-z$ and $z-x$ section from the stack at a plane containing the $\mathrm{Hf}$ atom. Pixel sizes are about $0.01 \mathrm{~nm}$ for the $\mathrm{x}$ - and $\mathrm{y}$-directions and $0.5 \mathrm{~nm}$ in the $\mathrm{z}$ direction, respectively. Reprinted from Ref. [6], with the permission of AIP Publishing.
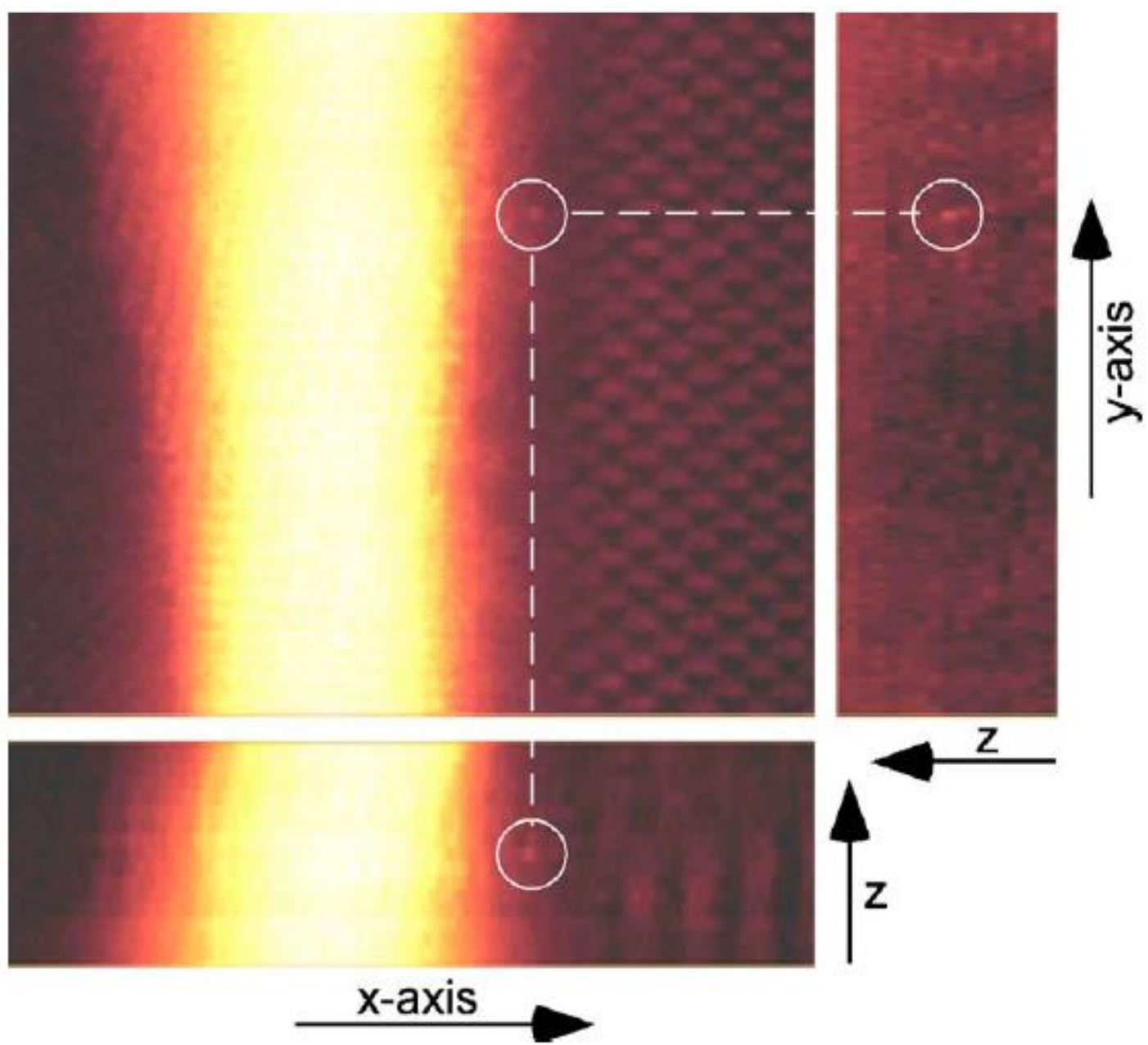
Figure 3. (a) An image from a $3 D$ focal series ADF STEM image stack from a sample of Pt nanoparticles supported on carbon black. (b) Two particles of size 8.3 (square) and $3.8 \mathrm{~nm}$ (circle) were selected. (b) Integrated intensity of both Pt particles as a function of defocus. The intensity from each particle is significant over a width of hundreds of nanometres. How wide this range is depends on both the size of the particle and also the semiangle of convergence. Solid line, $8.3 \mathrm{~nm}$ particle; dotted line, $3.8 \mathrm{~nm}$ particle. Taken from Ref. [8].

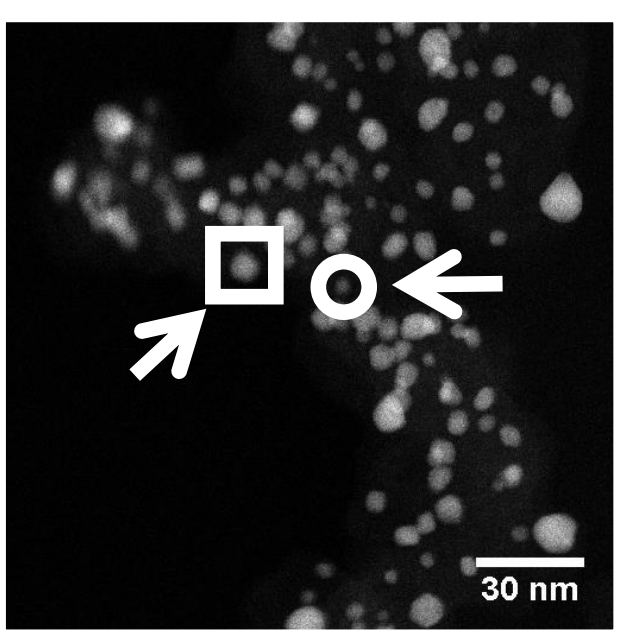

(a)

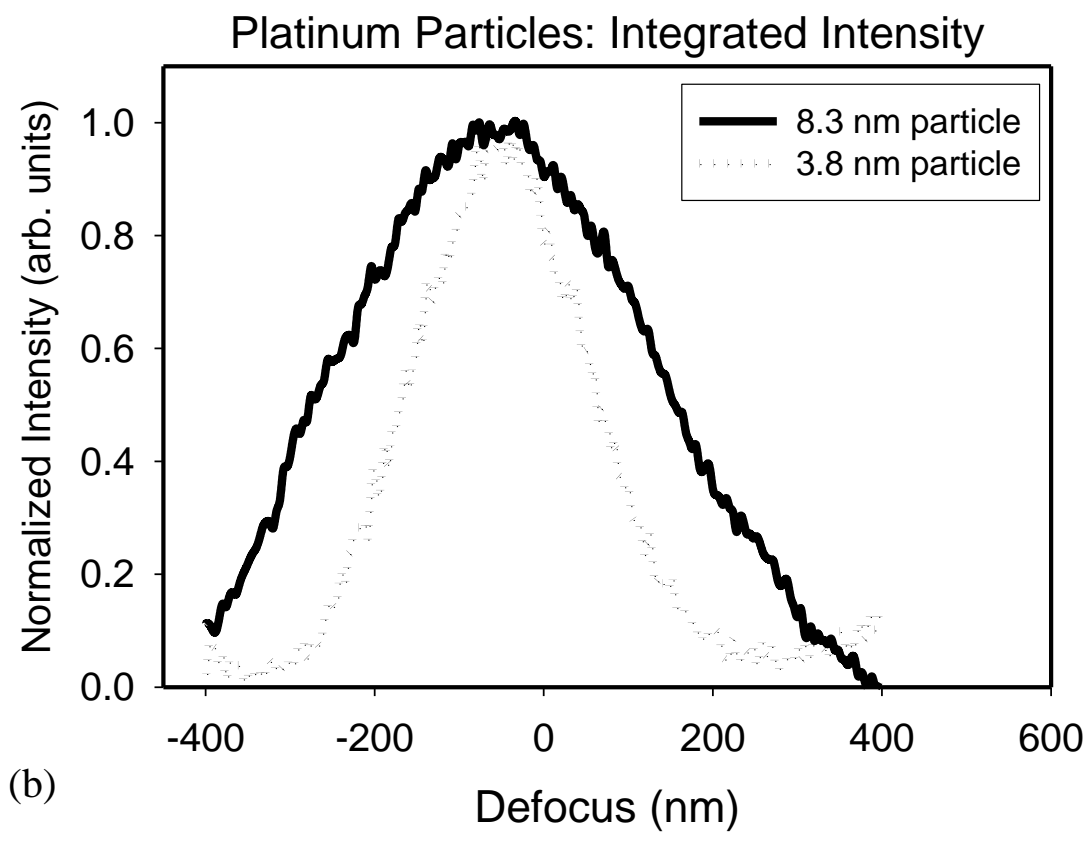


Figure 4. A section through the 3D OTF for ADF STEM. The large missing cone of transfer explains the depth elongation seen in Fig. 2. Taken from Ref. [8].

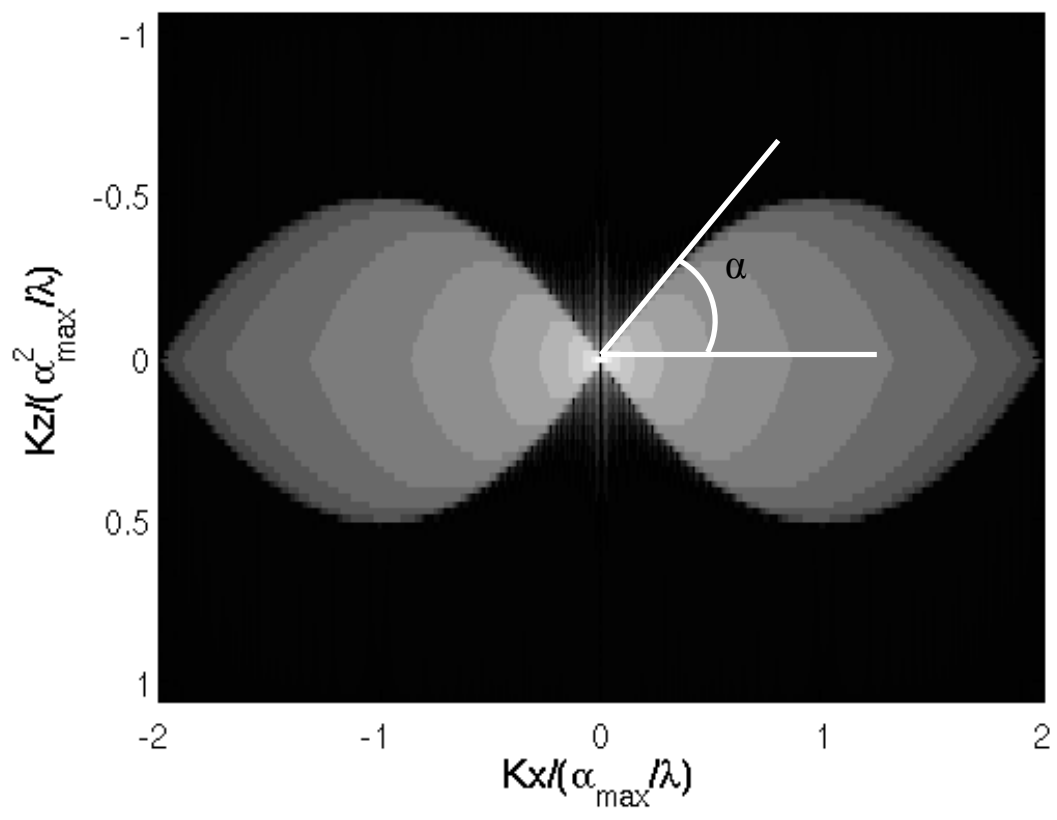


Figure 5. Intensity as a function of depth when imaging GaAs $<110>$ when the probe is located above the As column (solid line) and between columns (dotted line) for a defocus set at $5 \mathrm{~nm}$ deep into the crystal. Taken from Ref. [15].

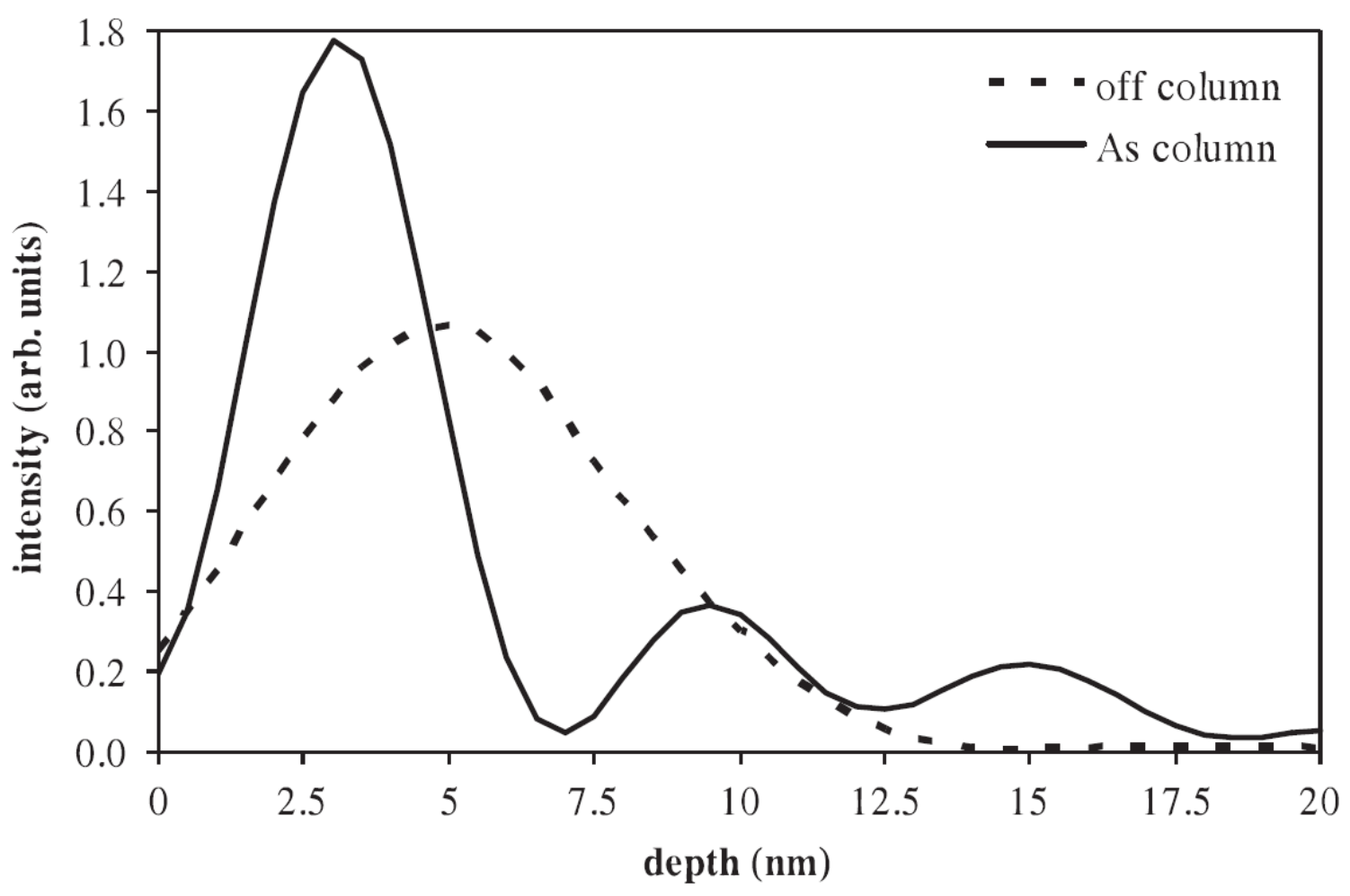


Figure 6. $x-z$ sections through a 3D ADF STEM image stack with imaging parameters $300 \mathrm{keV}, \alpha=23$ $\mathrm{mrad}, \mathrm{C} 3=-0.0778 \mathrm{~mm}$, and $\mathrm{C} 5=100 \mathrm{~mm}$. (a) A Hf atom at $\mathrm{z}=77 \AA$ in a-SiO ${ }_{2}$ matrix using the 3D linear imaging model. (b)-(e) Sections reconstructed from a through-focal series of 32 multislice images, ADF collection angle 75-250 mrad: (b) an individual $\mathrm{Hf}$ at $\mathrm{z}=77 \AA$ in $\mathrm{a}-\mathrm{SiO}_{2}$, (c)-(e) an individual hafnium atom replacing a silicon atom in $\langle 110\rangle$ c-Si at different depths. Dashed lines are the top and bottom surfaces of the sample. Black crosses are the $\mathrm{Hf}$ atoms. Black dots are the Si atoms. (f) Comparison of through-focal line profiles of an individual $\mathrm{Hf}$ atom in a-SiO $\mathrm{S}_{2}$ simulated by the multislice method and the 3D linear imaging model. Results from the 3D linear imaging model are scaled to match the maximum intensity of the multislice result. Images (b)-(d) are on the same vertical scale. Defocus is positive for underfocus. Depth is defined as defocus minus $-122.5 \AA$, which is the absolute defocus where the maximum intensity of the PSF appears. Reprinted from Ref. [16], with the permission of AIP Publishing
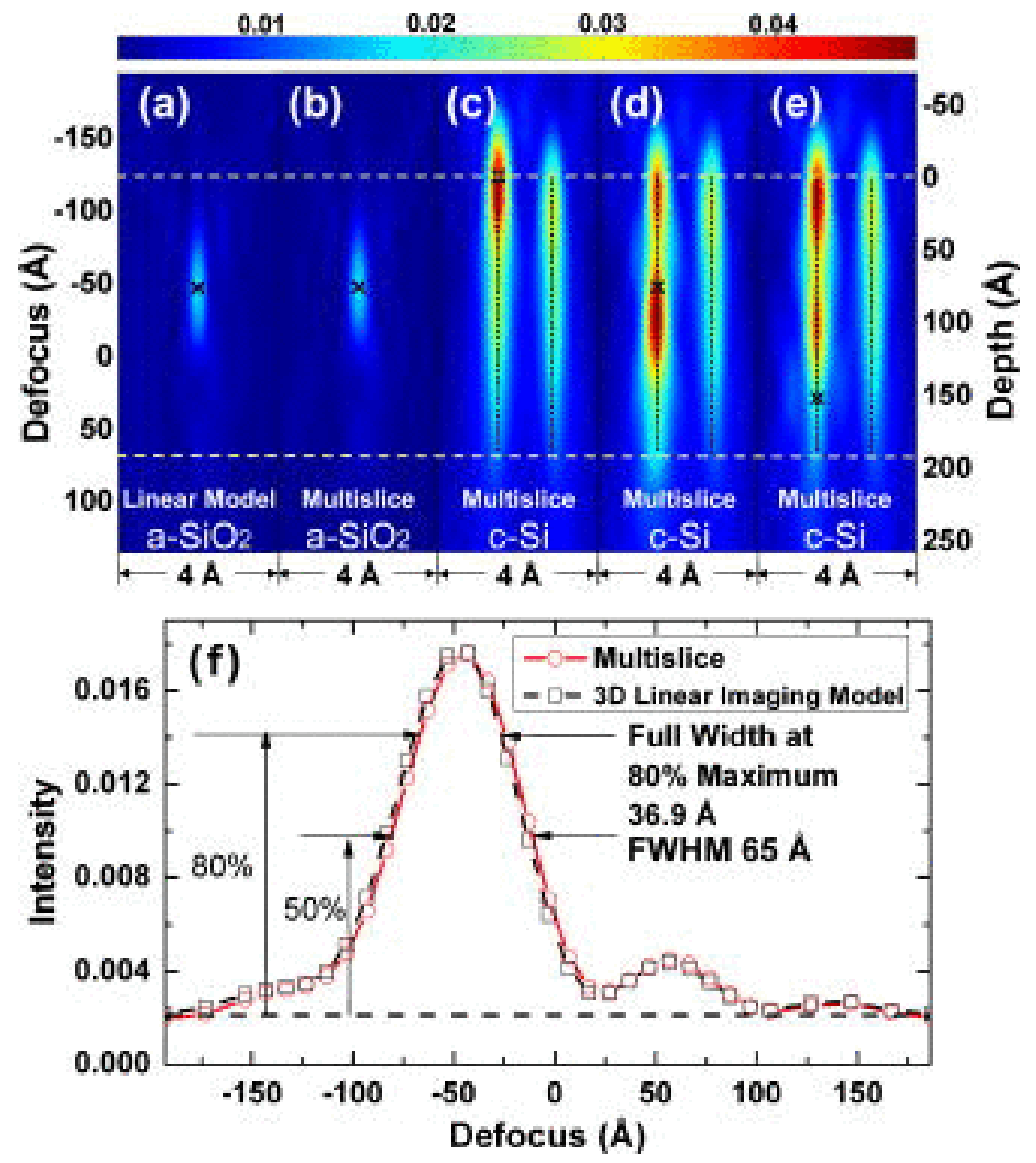
Figure 7. HR-STEM micrographs from part of a complete focal series of (a) and (b) two neighbouring mixed dislocations and (c) a pure edge dislocation. The position of the dislocations cores are marked with white crosses. (d) displays the experimentally measured rotation angle as a function of defocus for these three dislocations. The rotation is clockwise for one of the mixed dislocations and anticlockwise for the other. No significant rotation is observed for the case of the pure edge dislocation. Reprinted figure with permission from Ref.[24] copyright 2014 by the American Physical Society.

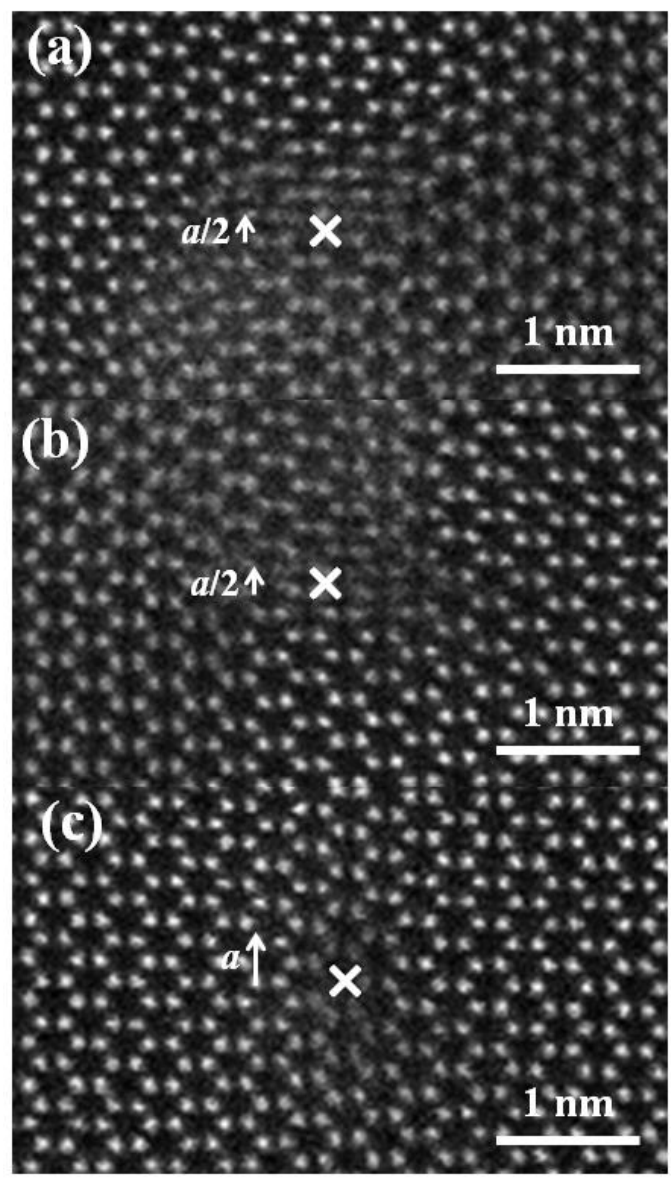

\section{(d) Rotation angle (degrees)}

$\begin{array}{lllllll}-0.8-0.6-0.4-0.2 & 0 & 0.2 & 0.4 & 0.6 & 0.8\end{array}$

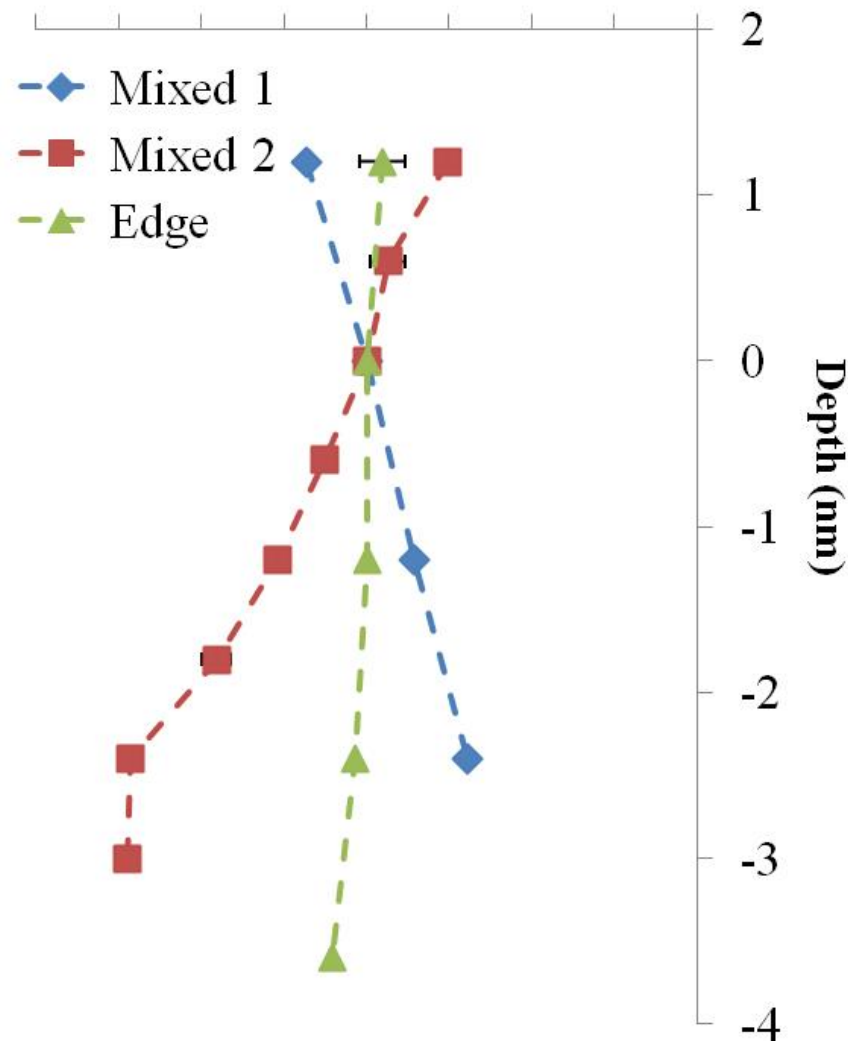


Figure 8. Through-focus series (a-c) showing the atomic configuration of a $\mathrm{Cd}$-core $90^{\circ}$ partial dislocation at defocus values of $10 \mathrm{~nm}, 11.2 \mathrm{~nm}$ and $15.2 \mathrm{~nm}$, respectively, indicating the presence of kinks along the dislocation line. The white dashed box indicates the associated extrinsic stacking fault. Taken from Ref. [26].
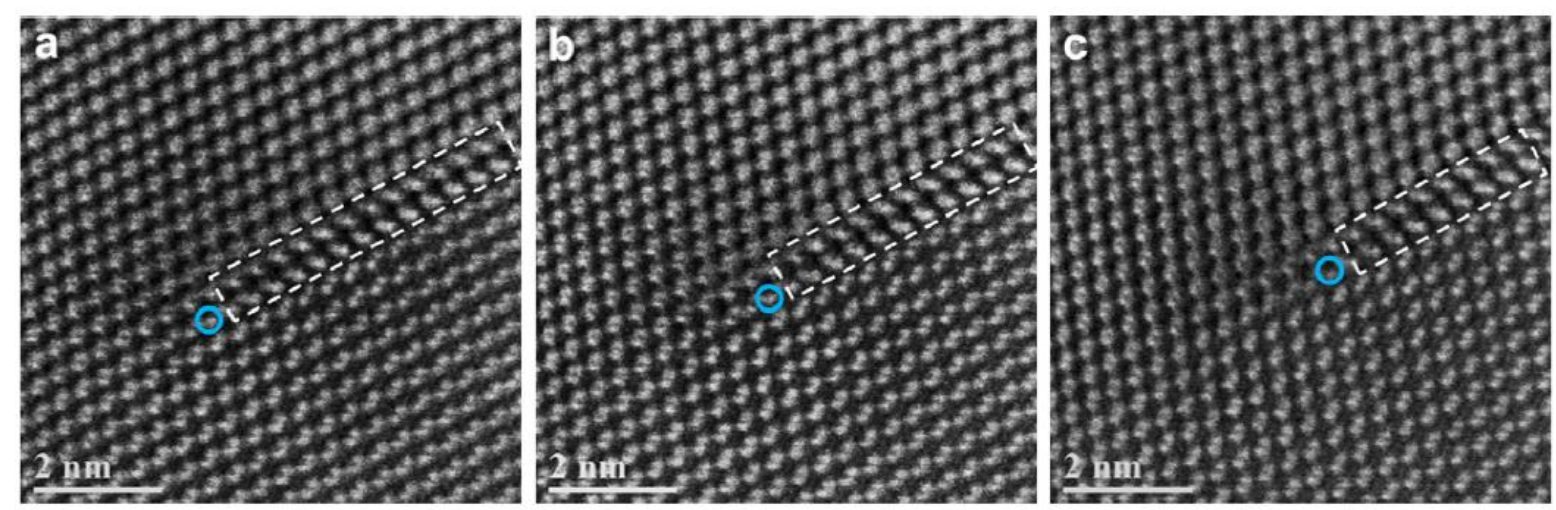
Figure 9. Experimental and simulated STEM-ADF image (inset) of the $1 / 2[\mathbf{a}+\mathbf{c}]+1 / 2[\mathbf{a}+\mathbf{c}]$ dissociated dislocation lying perpendicular to the electron beam. The experimental image is the average of 64 images (see supplementary information). The screw displacements associated with each of the partial dislocations can be observed, as indicated by the overlaid solid and dashed lines following the closer-to-focus stronger intensity peaks and further-from-focus weaker intensity peaks, respectively. A simulated image of the isotropic elastic model of a dissociated dislocation with a $1.65 \mathrm{~nm}$ dissociation distance is overlaid. The simulation was done with the beam focused $5 \mathrm{~nm}$ below the top entrance surface of a 10-nm thick foil. Taken from Ref. [28].

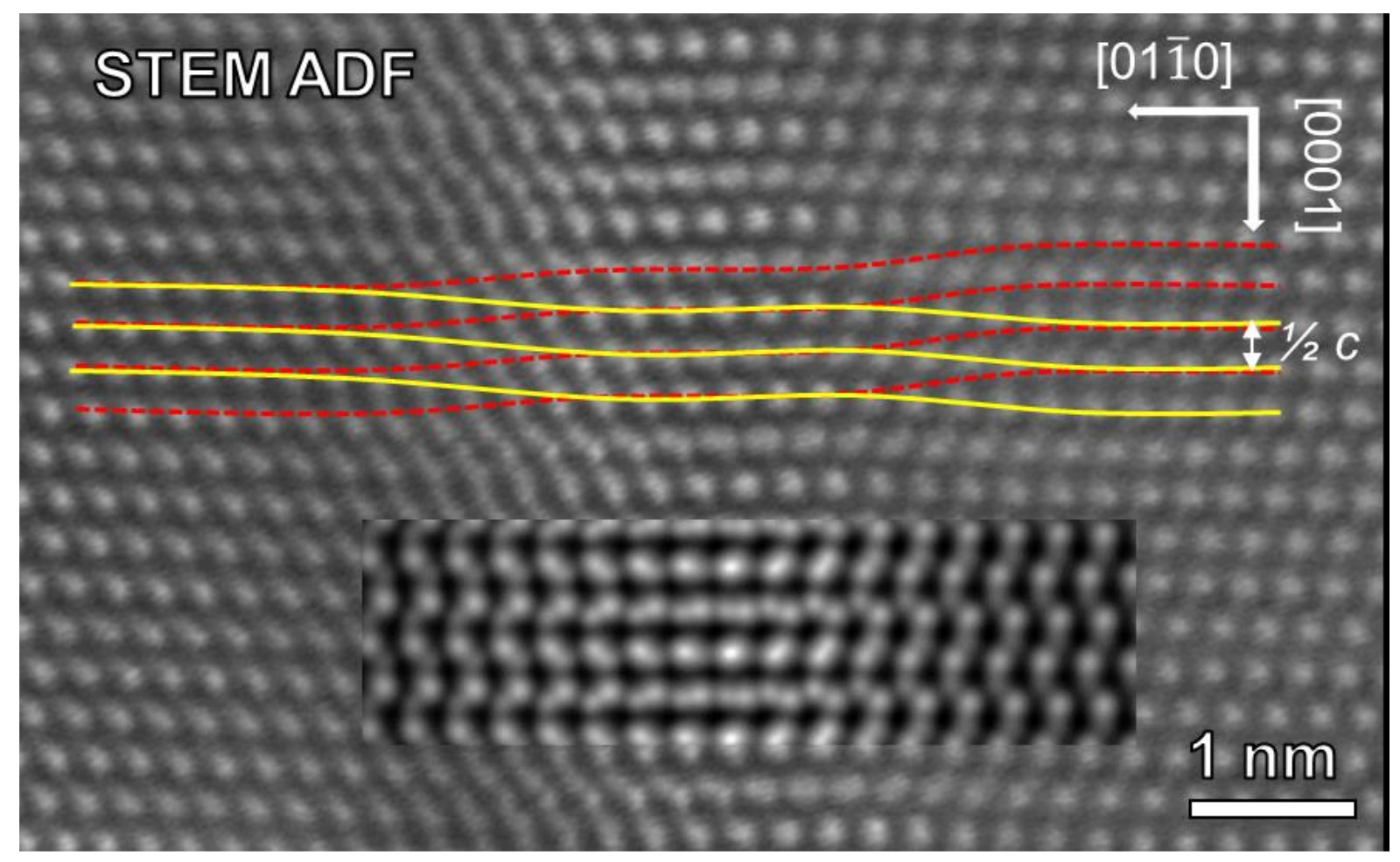

